\title{
The Effects of Milling Factors on Surface Finish of Chromium- Molybdenum Steel
}

\author{
Warachanin Wongsasiripat ${ }^{1}$ and Komson Jirapattarasilpls ${ }^{1, a}$ \\ ${ }^{1}$ Department of Production Technology Education, Faculty of Industrial Education and Technology, \\ King Mongkut's University of Technology Thonburi, Bangkok, Thailand
}

\begin{abstract}
This research was to study effect of milling, which was affected on surface finish of Chromium-Molybdenum steel. Fifty-four pieces of JIS SCM 415 steel was used specimen. The experiment was done by CNC vertical milling machine and cutting tool was PVD coated tungsten carbide tool. The factorial design was applied and factors were consisted of three factors: cutting speed and feed rate and depth of cut that were set in three levels. The results revealed that surface finish was affected by two main factors of feed rate and depth of cut. On the other hand, cutting speed was not significantly main factor. However, interaction between cutting speed, feed rate and depth of cut were factors controlling surface finish. Finally, the effects of milling factors on surface finish were analyzed and discussed.
\end{abstract}

\section{Introduction}

Chromium-Molybdenum steel is alloy steel that contains low carbon. This steel uses for auto part and some machinery such as gear, shaft and piston. Because of chromium composition between $1 \%$ and Molybdenum about $0.15 \%$ that steel have toughness and more tensile stress than other low carbon steel. To manufacturing of product, always this material was machined for milling process. This milling process was quality of dimension and surface finish that meet the needs of customer. Thus, factors of milling process could be affected to quality of machined surface. In previous study, factors of milling can be affected to quality of surface such as feed rate, cutting speed, depth of cut, cutting tool, cooling. The study of surface finish and effects of milling factors on surface finish have been report. The previous studies for surface finish of steel such as stainless steel [1], medium carbon steel [2-3] were studied. The others material has been studied such as titanium alloy [4-5], nickel alloy[56], aluminum alloy [7] and polymer [8]. They conclude that conditions of milling parameters based on feed rate, cutting speed, and depth of cut could be affecting to surface finish especially in case of steel. Therefore, Chromium-Molybdenum steel especially JIS SCM 415 steel was interested and effects of milling factors on surface finish for Chromium Molybdenum steel should be study.

\section{Material, Tool and Equipment}

Material used for this experiment was carbon steel that was grade JIS SCM 415. The properties of specimen were showed in Table 1. The specimens were prepared by dimension of $25 \mathrm{~mm} \times 50 \mathrm{~mm} \times 100 \mathrm{~mm}$. as shown in

\footnotetext{
a Corresponding author: komson.jir@kmutt.ac.th
}

Figure 1. The experiment was done by computerize numerically controlled milling machine: 'MIKRON model WF 21 D'. The cutting tool was tungsten carbide that coated by physical vapor deposition (PVD) as shown in Figure 2. The cutting tool was installed into tool holder that diameter was $16 \mathrm{~mm}$ as shown in Figure 3. The surface roughness tester 'Mitutoyo Model SJ-201' was used to measure surface finish.

Table 1. The Chemical Composition of JIS S50 C

\begin{tabular}{|c|c|}
\hline Element & \% wt. \\
\hline $\mathrm{C}$ & $0.13-0.18$ \\
\hline $\mathrm{Si}$ & $0.15-0.35$ \\
\hline $\mathrm{Mn}$ & $0.60-0.85$ \\
\hline $\mathrm{Cr}$ & $0.9-1.2$ \\
\hline $\mathrm{Mo}$ & $0.15-0.30$ \\
\hline
\end{tabular}

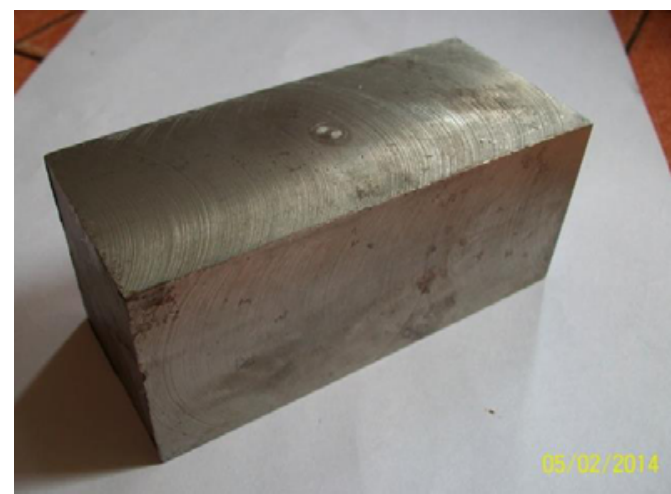

Figure 1. Specimen :JIS: SCM 415. 


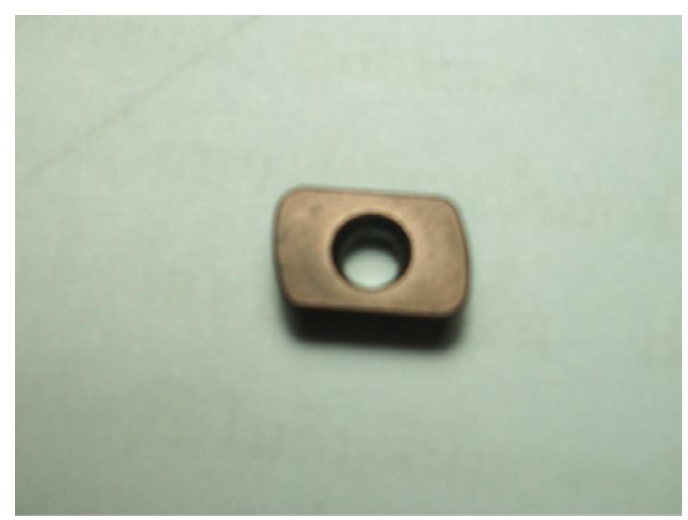

Figure 2. PVD coated tungsten carbide tool Specimen

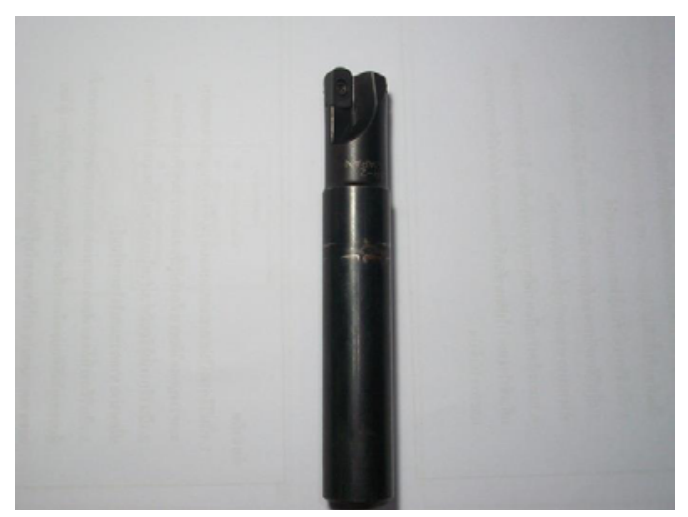

Figure 3. Tool Holder

\section{Experimental Procedure}

Experiment was applied by factorial design with experimental model of $3^{3}$. The actual experiment was randomly selected run by two replicates which 54 trials. Milling factors used for this experiment were cutting speed (v), feed rate (f) and depth of cut (d). Each factor was set at three levels. Cutting speeds (V) were 50, 100, and 150 meter per minute. Feed rate was set at 100, 200, and 300 millimeter per minute. Depth of Cut was 0.2, 0.4 and 0.6 millimeter. These factors were tested by pilot study before running the actual experiment.

The surface finishes of each specimen were measured by surface roughness average $(\mathrm{Ra})$. The measuring was done by the three times on machined surface. Data were analyzed by statistical methods to finding the factors affected to surface roughness.

\section{Results and Discussion}

The results of surface finish were observed and measured were showed as example in Figure 4. The initial setting factors to run for appropriate levels of milling were determined and surface roughness results were tested by the normality test. The test showed that the results were normality.

Effect of milling factors on surface finish was anylzed by the analysis of variance (ANOVA). ANOVA indicated that the effect of feed rate and depth of cut were significantly difference at the confidant level of .01. It means that feed rate and depth of cut were main factors that directly affected to surface finish. Furthermore, cutting speed was not main factor. However, interaction between each factor was found. That means interaction between three factors would be affect to surface roughness.

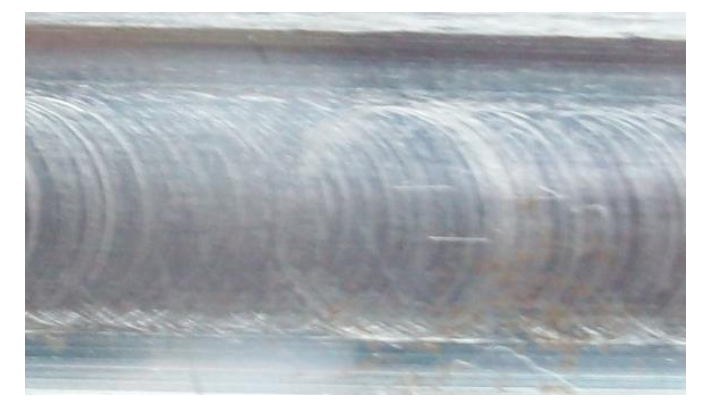

Figure 4. Surface Finish of Specimen Condition : $\mathrm{V}=50 \mathrm{~m} / \mathrm{min}, \mathrm{f}=300 \mathrm{~mm} / \mathrm{min} \mathrm{d}=0.20 \mathrm{~mm}$ $\mathrm{Ra}=1.861$

\subsection{The effects of feed rate and depth of cut on surface finish}

Feed rate and depth of cut were main factor affected to surface roughness, as shown ANOVA. The effect of feed rate was revealed that feed rate was higher, surface roughness was slightly up as shown in Figure 5. It means that the low feed rate was affected to good quality of surface. Meanwhile, the effect of depth of cut was shown in Figure 6. The increasing depth of cut was affected to enlargement of surface roughness. That means milling at higher depth of cut, it will be made poor quality of surface finish.

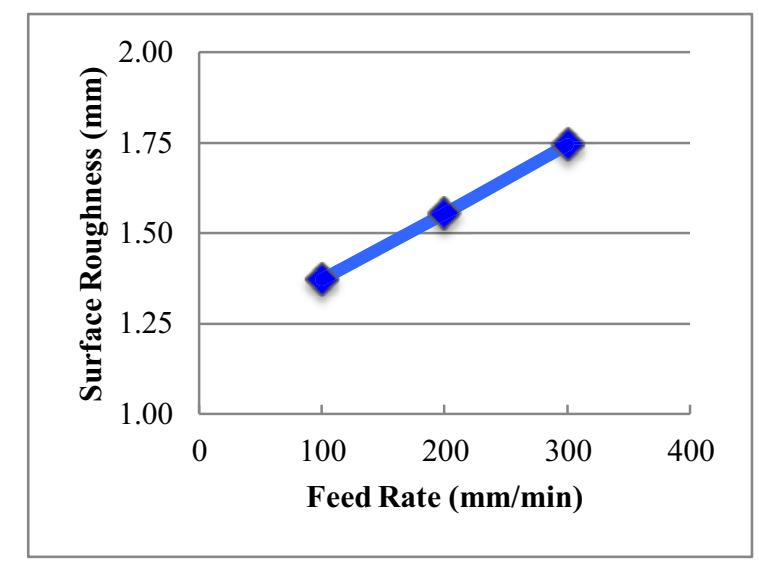

Figure 5. Effect of Feed Rate. 


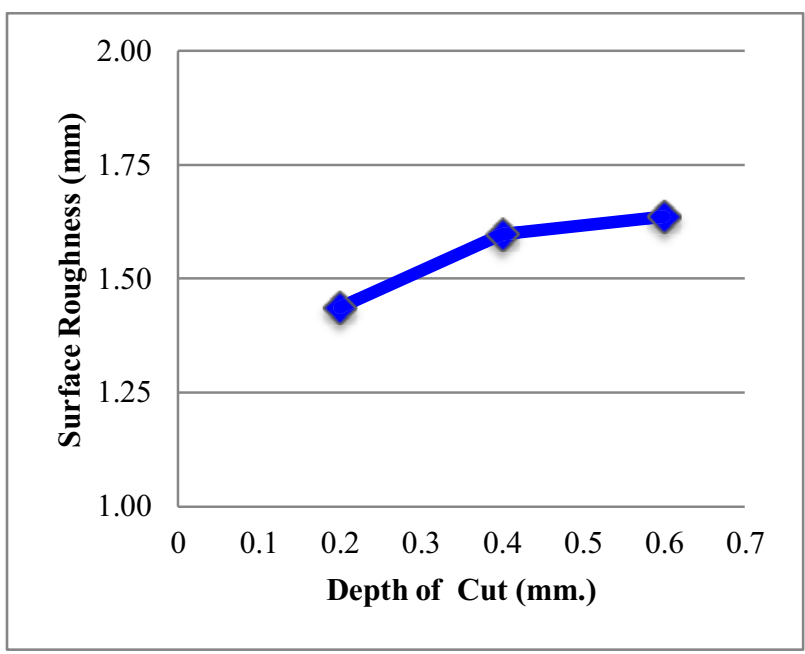

Figure 6. Effect of Depth of cut.

\subsection{The effects of interaction of milling factors on surface surface}

Since the cutting speed was low that the surface roughness was lowest when applied feed rate and depth of cut at low level as shown in Figure 6. As same as low cutting speed, medium cutting speed showed that using feed rate and depth of cut at low level could be produce best surface finish as showed in Figure 7. On other hand, at high cutting speed, surface finish was lowest at high depth of cut and low feed rate as showed in Figure 8. In order to good quality of surface finish, it was recommended to use low feed rate and use low cutting speed with low depth of cut but if it needs to use high cutting speed then use high depth of cut too.

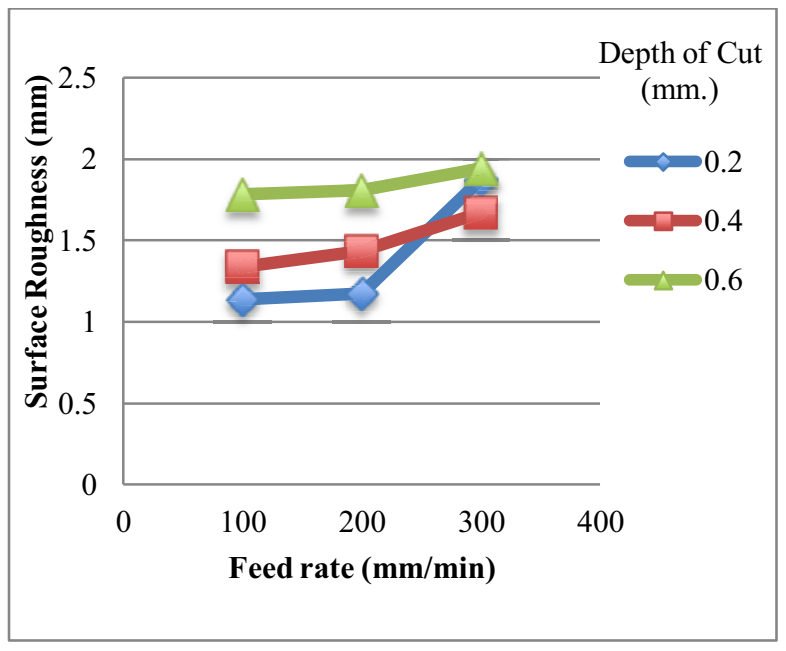

Figure 7. Effects of interaction of milling factors at Cutting speed $50 \mathrm{~m} / \mathrm{min}$

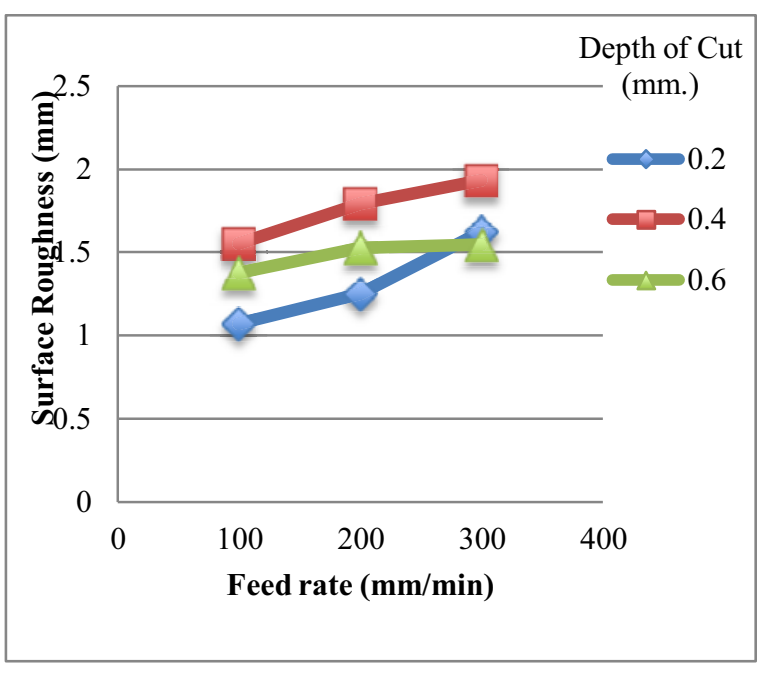

Figure 8. Effects of interaction of milling factors at Cutting speed $100 \mathrm{~m} / \mathrm{min}$

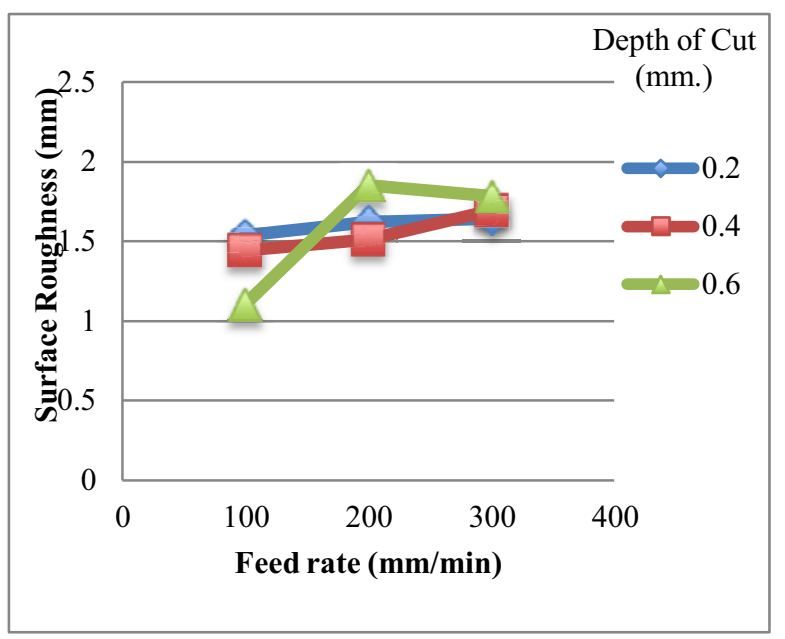

Figure 9. Effects of interaction of milling factors at Cutting speed $150 \mathrm{~m} / \mathrm{min}$

\section{Conclusion}

This research was experiment of effects of milling parameters on surface finish of Chromium-Molybdenum steel, JIS SCM 415, that was milling by coated tungsten carbide cutting tool. The results could be conclude that feed rate and depth of cut were main factors that affected to surface finish. Moreover, interaction of factors was found that interaction effects represent the combined effects of each factor on surface finish. The impact of cutting speed depends on the level of the feed rate and depth of cut. Moreover, the research was concluding as following;

- The low feed rate was affected to good surface finish better than high feed rate.

- The lower depth of cut will be made a good quality of surface finish more than higher. 
- The low cutting speed with low depth of cut and low feed rate can be produce good surface finish as same as cutting speed at high level with high depth of cut and low feed rate.

\section{References}

1. S.Luejanda and K.Jirapattarasilp, The Study of Surface Finish in Face Milling of Stainless Steel:AISI 304, Adv. Mater. Research, Vol. 650 (2013), p.606.

2. P.Chutrakul, K.Jirapattarasilp, The Effect of Hard Milling on Surface Finish of Medium Carbon Steel, Adv. Mater. Research, Vol. 904 (2014), p.213.

3. R.J.Wang, Y. L.Zhang, H.B.Liu, M.H.Du, Surface Finish Study of Hard Milling 45 Steel with PCBN ToolAdv. Mater. Research, Vol. 468-471 (2012), p. 1467.

4. K.Tsuda, K.Okuda, H. Shizaka, and M. Nunobiki, $A$ Study of the Micro End Milling of Titanium Alloy, Adv. Mater. Research, Vol 325(2011), p.588.

5. D.Ulutan,T. Ozel, Machining induced surface integrity in titanium and nickel alloys: A review International Journal of Machine Tools and Manufacture, Vol.51 (3) (2011), p. 250.

6. M.A.Hadi, J.A.Ghani, C.A.Haron, N.A. Raof, Tribological Characterization of the Cutting Zone in Milling Nickel Based Alloy, Appl. Mech. and Mater. Vols. 554 (2014) p. 12

7. J.Abbas, A.A.Habaibeh, and D. Z.Su, The Investigation of Prediction Surface Roughness from Machining Forces in End Milling Processes, Key Eng. Mater. ,Vol.486, (2011), p 91.

8. S.Obma and K.Jirapattarasilp, The Effect of Vertical Milling on Finished Surface of Polymethylmethacrylate Sheet, Appl. Mech. and Mater. Vols. 157-158 (2012) p.263 